\title{
Eine Bemerkung über die Räume vom Typus $(F)$
} von

\author{
M. EIDELHEIT und S. MAZUR (Liwów).
}

In dieser Note zeigen wir vorerst $\left.{ }^{1}\right)$ :

1. In jedem Raume $E$ vom Typus $(F)$ kann man eine mit der ursprünglichen Metrik $(x, y)$ äquivalente Metrik $(x, y)^{*}$ einführen, so da $\beta$ stets $(x, y)^{*}=(x-y, 0)^{*}$ gilt und $d a \beta(t x, 0)^{*}$ für $x \neq 0$ als Funktion der reellen Veränderlichen $t \geqslant 0$ monoton wachsend ist.

Mit Benutzung dieser Bemerkung ergibt sich alsdann leicht der folgende von Herrn S. Banach ohne Beweis angegebene Satz ${ }^{2}$ ):

2. Damit ein Raum $E$ vom Typus $(F)$ endlichdimensional sei, ist notwendig und hinreichend, $d a \beta$ es in ihm eine offene kompakte Menge gebe.

Zum Beweis von 1 setzen wir

$$
(x, y)_{w}=\sup _{0 \leqq s \leqq w}(s x, s y)
$$

für $x, y \in E$ und rationale $w>0$. Bei festem $w$ bildet ersichtlich $(x, y)_{w}$ eine mit $(x, y)$ äquivalente $\left.{ }^{8}\right)$ Metrik in $E$; ferner ist $(x, y)_{w}=(x-y, 0)_{w}$ und

$$
\left(t_{1} x, 0\right)_{w} \leqslant\left(t_{2} x, 0\right)_{w} \text { für } 0 \leqslant t_{1}<t_{2} .
$$

1) Wegen der hier benutzten. Terminologie vgl.: S. Ban a ch, Théorie des opérations linéaires, Warszawa 1932.

3 ) L. c. '), p. 236; für den Fall der Räume vom Typus $(B)$ wurde dieser Satz von Herrn F. Riesz bewiesen: F. Riesz, Uber lineare Funktionalgleichungen, Acta math. 41 (1918) p. $71-98$.

3) Hier, wie auch im Folgenden, wird die Tatsache benutzt, das die Multiplikation der Zahlen mit Elementen stetig ist; vgl.: S. Mazur und W. Orlic z, Über Folgen linearer Operationen, Studia Math. 4 (1933) p. 152-157. 
Wir ordnen nun alle positiven rationalen Zahlen $w$ in eine Folge $\left\{w_{n}\right\}$ und setzen

$$
(x, y)^{*}=\sum_{n=1}^{\infty} \frac{1}{2^{n}} \frac{(x, y)_{w_{n}}}{1+(x, y)_{u_{n}}}
$$

für $x, y \in E ;$ da $(x, y)_{w_{n}}$ eine mit $(x, y)$ äquivalente Metrik in $E$ bildet, so gilt dasselbe bekanntlich auch für $(x, y)^{*}$. Außerdem ist $(x, y)^{*}=(x-y, 0)^{*}$ und mit Rücksicht auf (2)

$$
\left(t_{1} x, 0\right)^{*} \leqslant\left(t_{2} x, 0\right)^{*} \text { für } 0 \leqslant t_{1}<t_{2} .
$$

Es bleibt noch zu zeigen, $\mathrm{daß}$ in (3) die Gleichheit nur für $x=0$ stattfindet; wir können uns dabei offenbar auf rationale $t_{1}, t_{2}$ beschränken. Aus $\left(t_{1} x, 0\right)^{*}=\left(t_{2} x, 0\right)^{*}$ ergibt sich, indem man wieder (2) anwendet, $\left(t_{1} x, 0\right)_{u_{n}}=\left(t_{2} x, 0\right)_{x u_{n}}$, d. h. $\left(t_{1} x, 0\right)_{w}=\left(t_{2} x, 0\right)_{w}$ für rationale $w>0$; setzen wir hier der Reihe nach $w=1, t_{1}^{1} t_{2}^{-1}, \ldots, t_{1}^{n} t_{2}^{-n}, \ldots$, so kommt sofort wegen (1)

$$
\sup _{0 \leqq s \leqq t_{2}}(s x, 0)=\sup _{0 \leqq s \leqq t_{1}^{n} t_{2}^{n+1}}(s x, 0) ;
$$

daraus folgt aber $\sup _{0 \leqq s \leqq t_{2}}(s x, 0)=0$, da $t_{1}^{n} t_{2}^{-n+1} \rightarrow 0$, und mithin auch $x=0$, wie behauptet.

Wir kommen nun zu dem Beweis von 2. Die Notwendigkeit kann in derselben Weise, wie im speziellen Falle der Räume vom Typus $(B)$ gefolgert werden; bilden die Elemente $e_{1}, e_{2}, \ldots, e_{m}$ eine Basis in $E$ und ordnen wir jedem $x=t_{1} e_{1}+t_{2} e_{2}+\ldots+t_{m} e_{m}$ mit reellen $t_{k}$ das $m$-tupel $\left(t_{1}, t_{2}, \ldots, t_{m}\right) z u$, so ist dadurch eine isomorphe Abbildung von $E$ auf den $m$-dimensionalen euklidischen Raum gegeben. Um die Hinlänglichkeit zu erkennen, beachten wir, daß es nach 1 keine Beschränkung der Allgemeinheit bedeutet, wenn wir voraussetzen, $\mathrm{da} B(t x, 0)$ für $x \neq 0$ als Funktion der reellen Veränderlichen $t \geqslant 0$ monoton wachsend ist. Bei passendem $r>0$ ist die Menge aller $x \in E$ mit $(x, 0)<3 r$ kompakt; bezeichnen wir nun mit $K$ die $\operatorname{Kugel}(x, 0) \leqslant r$, so gilt der Hilfssatz: Ist $A$ eine lineare, abgeschlossene und echte Teilmenge von $E$, so gibt es ein $x_{0} \in K$ mit $\left(x_{0}, a\right) \geqslant r$ für $a \in A$. Zum Beweise setzen wir $(x, A)=\inf _{x \in A}(x, a)$ für $x \in E, Q=\sup _{x \in K}(x, A)$. Ist $x_{n} \in K,\left(x_{n}, A\right) \rightarrow \varrho$ und die Teilfolge $\left\{x_{n_{k}}\right\}$ gegen $x_{0} \in E$ kon- vergent, so ist $x_{0} \in K$ und $\left(x_{0}, A\right)=\varrho$, da einerseits trivialerweise $\left(x_{0}, A\right) \leqslant 0$, andererseits wegen $\left(x_{n_{k}}, a\right) \geqslant\left(x_{n_{k}}, A\right)$ auch $\left(x_{0}, a\right) \geqslant \rho$ für $a \in A$, d. h. $\left(x_{0}, A\right) \geqslant \varrho$ gilt; weil außerdem $\left(x_{0}, A\right) \leqslant\left(x_{0}, 0\right)$ und folglich $\varrho \leqslant r$ gilt, so kommt es nur darauf an, die Ungleichung $\varrho<r$ auszuschließen. Wir bemerken zunächst, $\mathrm{da} ß$ es für jedes $\bar{x} \in K$ ein $\bar{a} \in A$ mit $(\bar{x}, \bar{a})=(\bar{x}, A)$ gibt; denn ist $a_{n} \in A$, $\left(\bar{x}, a_{n}\right) \rightarrow(\bar{x}, A)$, so gilt sicherlich $\left(a_{n}, 0\right)<3 r$ für alle hinreichend großen Indizes $n$, in Anbetracht dessen, $\mathrm{daß}\left(a^{n}, 0\right) \leqslant\left(\bar{x}, a_{n}\right)+(\bar{x}, 0)$; ist die Teilfolge $\left\{a_{n_{k}}\right\}$ gegen $\bar{a} \in E$ konvergent, so leistet $\bar{a}$ das Verlangte. Insbesondere also gibt es ein $a_{0} \in A$ mit $\left(x_{0}, a_{0}\right)=0$; wäre $\Leftrightarrow<r$, so wäre noch $x_{1}=s\left(x_{0}-a_{0}\right) \in K$ bei passendem $s>1$ und es gäbe wieder ein $a_{1} \in A$ mit $\left(x_{1}, a_{1}\right)=\left(x_{1}, A\right)$; da offenbar $\varrho>0$ und mithin $x_{0}-a_{0}-\frac{1}{s} a_{1} \neq 0$, so erhielte man dann

$$
\begin{gathered}
\left(x_{1}, a_{1}\right)=\left(s\left(x_{0}-a_{0}-\frac{1}{s} a_{1}\right), 0\right)>\left(x_{0}-a_{0}-\frac{1}{s} a_{1}, 0\right) \\
=\left(x_{0}, a_{0}+\frac{1}{s} a_{1}\right) \geqslant\left(x_{0}, A\right), \text { d. h. }\left(x_{1}, A\right)>\varrho
\end{gathered}
$$

was gegen die Definition von $\varrho$ verstößt. Damit ist der Hilfssatz bewiesen und von hier aus kann man schon ähnlich wie im Falle der Räume vom Typus $(B)$ vorgehen. Angenommen, der Raum $E$ sei unendlichdimensional. Wir erklären mittels Induktion die Elemente $e_{n} \in E$ folgenderweise : $1^{\circ}$ Sei $e_{1} \in K ; 2^{\circ}$ sind $e_{1}, e_{2}, \ldots, e_{m}$ bereits erklärt, so bilden alle Elemente $a=t_{1} e_{1}+t_{2} e_{2}+\ldots+t_{m} e_{m}$ mit reellen $t_{k}$ eine lineare, abgeschlossene (sogar mit einem euklidischen Raume isomorphe) und echte Teilmenge $A$ von $E$; es gibt also ein $e_{m+1} \in K$ mit $\left(e_{m+1}, a\right) \geqslant r$ für $a \in A$. Wegen $\left(e_{p}, e_{q}\right) \geqslant r$ für $p \neq q$ enthält die Folge $\left\{e_{n}\right\}$ keine konvergente Teilfolge und dies stelit mit $e_{n} \in K$ im Widerspruch.

(Reçu par la Rédaction le 2. 12. 1937). 